CERN-TH/99-398

hep-th/9912147

\title{
D-Branes on K3-Fibrations
}

\author{
P. Kaste, W. Lerche, C.A. Lütken ${ }^{1}$ and J. Walcher 2 \\ Theory Division, CERN, Geneva, Switzerland
}

\begin{abstract}
$B$-type $D$-branes are constructed on two different $K 3$-fibrations over $\mathbb{P}_{1}$ using boundary conformal field theory at the rational Gepner points of these models. The microscopic CFT charges are compared with the Ramond charges of $D$-branes wrapped on holomorphic cycles of the corresponding Calabi-Yau manifold. We study in particular $D 4$-branes and bundles localized on the $K 3$ fibers, and find from CFT that each irreducible component of a bundle on $K 3$ gains one modulus upon fibration over $\mathbb{P}_{1}$. This is in agreement with expectations and so provides a further test of the boundary CFT approach to $D$-brane physics.
\end{abstract}

CERN-TH/99-398

December 1999

1 On leave from Dept. of Physics, University of Oslo, N-0316 Oslo, Norway

2 Also at Institut für Theoretische Physik, ETH-Hönggerberg, CH-8093 Zürich, Switzerland 


\section{Introduction}

In the 'large radius' region of moduli space, strings can be viewed as moving in a fixed extrinsic background space-time whose geometry is determined by the closed string sector of the theory itself; in particular it must be a Calabi-Yau (CY) space. At string tree level these classical geometric data and some of the quantum corrections are encoded in $N=2$ superconformal field theory on the plane, hereafter called 'bulk' CFT. At special points in its moduli space the CFT is rational and can therefore be exhaustively studied using Gepner's construction. The complementary viewpoints provided by the 'intrinsic, microscopic' CFT picture, and the 'extrinsic, macroscopic' CY sigma-model, have provided a wealth of information about the closed string sector, most notably the phenomenon of mirror symmetry, amounting to a profound redirection of our thinking about the structure of space-time at small scales.

Our understanding of the open string sector is currently undergoing a parallel evolution which may ultimately provide a similar redirection of our thinking about the gauge structure of the theory. Indeed, a major effort is currently underway to extend the mirror map to the gauge bundles that may decorate various cycles in CY manifolds (see e.g., [1,2]).

For the open string sector the appropriate microscopic description is provided by boundary conformal field theory on the upper half plane (BCFT), and the corresponding macroscopic objects are $D$-branes wrapped on cycles of the CY target spaces embodied in the closed string sector. Since open strings can end on $D$-branes, these should be intimately related to the boundary states of the (microscopic) BCFT. In fact they are supposedly nothing but manifestations of the boundary states at large radii of the compactification manifold, where naive geometrical concepts apply. On the other hand, at small radii instanton corrections can swamp out any classical picture, to an extent where the very notion of space-time, as well as the vector bundles it supports, evaporates.

However, it is conceivable that the microscopic BCFT still encodes some of the geometric information pertaining to the $D$-branes at large radii, like the intersection properties of the cycles around which they wrap. This can be tested at the "Gepner" points in moduli space where the theory is exactly solvable, and thus opens a window on $D$-brane physics in a regime where quantum corrections are strong. This approach has been pioneered in refs. [3 6], building on earlier work, for example [7 10]. 
Our purpose in this letter is to apply the methods developed in these papers to study properties of $D$-branes on $K 3$-fibrations. The motivation is twofold. First, $K 3$-fibrations are important in the context of heterotic-type II duality [11], and their quantum algebraic geometry is well understood. In particular, for some cases the periods, monodromy and analytic continuation to Gepner points are either directly available from the literature [12], or can be obtained with a reasonable amount of effort. Second, for generic threefolds the quantum corrections at the Gepner point are strong, and for some states [3-6] there is no good match between BCTF data at the Gepner point and geometrical $D$-brane data at large radii - for these cases it may not always be clear whether the quantum geometries really differ so much, or whether the method fails. However, for $K 3$ fibrations we may expect that some quantities are protected by non-renormalization properties of the $K 3$, while the whole theory is not as trivial as if we would be looking just at an isolated $K 3$. Therefore, studying $K 3$-fibrations might not only offer new insights in $D$-brane physics at small radii, but also provide further tests of the validity of the BCFT method.

In Sect. 2, we review the BCFT approach at the Gepner point of generic $n$-folds, but also improve and clarify some steps in the papers [3-6]. Where our work overlaps, we are in agreement, and we explain why this is so. In Sect. 3 we then discuss some basic $D$-geometric features of the two $K 3$-fibrations we study. In section four, we compare the BCFT and the geometrical data, producing lists of $D$-brane charges that correspond to the CFT boundary states. Finally, in Sect. 4 we discuss some features of these data and find agreement with expectations.

\section{Gepner models and boundary conformal field theory}

The Gepner construction is a GSO-projected tensor product of $r$ minimal models at levels $k_{i}$, whose central charges $c_{i}=3 k_{i} /\left(k_{i}+2\right)$ add up to $3 n(n=1,2,3, \ldots)$, suitably twisted to enforce modular invariance of the partition function. An explicit connection with the geometric data at 'large radius' is provided by the Landau-Ginzburg representation of minimal models, provided that the tensor product is extended by a trivial factor $k_{r+1}=0$ when $n+r$ is odd. Let $K=\operatorname{lcm}\left\{h_{1}, \ldots, h_{r}\right\}$, where $h_{i}=k_{i}+2$ are called the heights of the minimal models. The $w_{i}=K / h_{i}$ will here be called the (relative) weights of the minimal models in the given product. Since $K$ is even when $n+r$ is odd, $h_{r+1}=2$ is automatically a divisor of $K$ and the weight of the trivial extension is $w_{r+1}=K / 2$. The smooth $\mathrm{CY} n$-fold is then obtained by desingularizing 
the degree $K$ quasi-homogeneous hypersurface in the weighted projective space of dimension $\operatorname{dim}(w)-1$ with scaling weights $w_{i}$.

We consider here only the two Gepner models $10^{2} 4^{2} 0 / \mathbb{Z}_{12}=10 \otimes 10 \otimes 4 \otimes 4 \otimes 0 / \mathbb{Z}_{12}$ and $6^{2} 2^{3} / \mathbb{Z}_{8}$. By the above algorithm, the first model contains the CY manifold which is defined classically by resolving the singularities of a quasi-homogeneous polynomial hypersurface of total degree 12 in a four-dimensional projective space weighted by $(1,1,2,2,6)$, hereafter denoted $\mathbb{P}(1,1,2,2,6)[12]$ for short. The second one contains the geometric data for a degree 8 projective variety, denoted $\mathbb{P}(1,1,2,2,2)[8]$ for short. Both these varieties are $K 3$-fibrations over $\mathbb{P}_{1}$. Their quantum geometry has been extensively investigated using mirror symmetry [12,13], and we will draw heavily on this information.

Open string states satisfying Neumann or Dirichlet boundary conditions are built from generalized coherent states (Ishibashi states) of a boundary conformal field theory. The coherent basis $|i\rangle\rangle_{\Omega}=\sum_{n}|i, n\rangle \otimes U_{\Omega} \overline{|i, n\rangle}$ is obtained by tracing over the modules labeled by the primary fields $|i\rangle=|i, 0\rangle$. The operator $U_{\Omega=A, B}$ selects socalled A- or B-type states by enforcing charge relations between the 'left' and 'right' sectors. Acceptable string states must satisfy Cardy's modular consistency conditions, in this case on the annulus. For A-type states Cardy's solutions $\| i\rangle\rangle_{A}=$ $\left.\sum_{j} S_{i j} /\left(S_{0 j}\right)^{1 / 2}|j\rangle\right\rangle_{A}$ apply. They are built from the $S$-matrices, which for minimal models at height $h$ (whose primary fields are labelled $|i\rangle=|l, m, s\rangle$ ), are simple trigonometric functions, whose $S U(2)_{k}$ part is just $S_{l, l^{\prime}}=\sqrt{2 / h} \sin \left(\pi(l+1)\left(l^{\prime}+1\right) / h\right)$. The construction can be adapted to B-type states by restricting the sum to run over labels appropriate for this case, and extended to Gepner models by tensoring such minimal model states $[7,8]$. Consequently, every detail about these classes of rational boundary states in Gepner models is known. A- and B-type boundary states $\| L M S\rangle\rangle_{\Omega}$ are labeled by vectors $L, M$ of $S U(2)$ quantum numbers from the minimal models in the tensor product, as well as a vector $S$ which distinguishes the NS- and R-fields. We shall return to the classification of boundary states in Gepner models elsewhere, since this will be important in establishing a complete correspondence between non-perturbative string theory and the geometry of $D$-branes in CY spaces.

The microscopic version of the intersection numbers in classical geometry should be 'overlap integrals' (open string amplitudes) between boundary states $\| L M S\rangle\rangle_{\Omega}$ and $\| \widetilde{L} \widetilde{M} \widetilde{S}\rangle\rangle_{\Omega}$. As explained in ref. [3] these can be computed by evaluating the 
Witten index in the Ramond sector. The result depends on the parity of $n+r$. When $n+r$ is even the intersection matrix for B-type boundary states is:

$$
I^{B e}={ }_{B}\left\langle\left\langle\widetilde{L} \widetilde{M} \widetilde{S}\left\|I^{e}\right\| L M S\right\rangle\right\rangle_{B}=C_{e} \prod_{i=1}^{r} \sum_{l_{i}=1}^{2 h_{i}} \delta_{l / 2,(\widetilde{M}-M) / 2}^{(K)} \prod_{j=1}^{r} N_{L_{j} \widetilde{L}_{j}}^{l_{j}},
$$

where $C_{e}(S-\widetilde{S})$ is a known 'constant', $M=\sum_{j} w_{j} M_{j}=1, \ldots, 2 K, l=\sum_{j} w_{j} l_{j}$, and the $S U(2)_{k}$ fusion coefficients are given by the Verlinde formula $N_{L \widetilde{L}}^{l}=$ $\sum_{l^{\prime}} S_{l^{\prime}, L} S_{l^{\prime}, l} S_{\widetilde{L}, l^{\prime}}^{*} / S_{l^{\prime}, 0}$. The clever trick [3] is to extend the definition of the fusion coefficients in a formal but natural way (compatible with the Verlinde formula) so that they become periodic over the extended range of labels. By virtue of the properties of the S-matrices and the Verlinde formula we have

$$
N_{L, \widetilde{L}}^{l}=\left\{\begin{aligned}
1 & \text { for } l=|L-\widetilde{L}|,|L-\widetilde{L}|+2, \ldots,(L+\widetilde{L}) \\
-1 & \text { for } l=-|L-\widetilde{L}|-2,-|L-\widetilde{L}|-4, \ldots,-(L+\widetilde{L}+2) \\
0 & \text { otherwise }
\end{aligned}\right.
$$

Eq.(11) coincides with the formula derived in ref. [3]. When $n+r$ is odd, we find a similar expression

$$
\begin{aligned}
I^{B o} & ={ }_{B}\left\langle\left\langle\widetilde{L} \widetilde{M} \widetilde{S}\left\|I^{o}\right\| L M S\right\rangle\right\rangle_{B} \\
& =C_{o} \prod_{i=1}^{r} \sum_{l_{i}=1}^{2 h_{i}}(-1)^{(M-\widetilde{M}+l) / K} \delta_{l / 2,(\widetilde{M}-M) / 2}^{(K / 2)} \prod_{j=1}^{r} N_{L_{j} \widetilde{L}_{j}}^{l_{j}},
\end{aligned}
$$

but with half the period in the $\delta$-function and an additional sign factor.

Gepner models enjoy a large group of discrete symmetries which play a fundamental role in both the structure of the CFT and its geometrical limits. Since the intersection matrices are computed at the Gepner points they inherit many of these symmetries. This can be exploited to give a much simpler representation of the intersection matrix in terms of two (highly reducible) representations of the abelian group $\mathbb{Z}_{K}$. Define the fundamental $K$-dimensional 'shift' matrices:

$$
\gamma_{ \pm}(K)=\left(\begin{array}{cccccc}
0 & 1 & 0 & \cdots & 0 & 0 \\
0 & 0 & 1 & \cdots & 0 & 0 \\
\vdots & \vdots & \vdots & \ddots & \vdots & \vdots \\
0 & 0 & 0 & \cdots & 0 & 1 \\
\pm 1 & 0 & 0 & \cdots & 0 & 0
\end{array}\right)_{K \times K}
$$


and note that $\gamma_{+}(K)$ has period $K$ while $\gamma_{-}(K)$ has period $2 K$, because $\gamma_{ \pm}(K)^{K}=$ \pm 1 . For fixed values of $L, \widetilde{L}$ the intersection form can be regarded as a matrix with rows and columns labelled by $\widetilde{M}$ and $M$. When $n+r$ is even we find that $I^{B}$ is most simply expressed as a polynomial in $g=\gamma_{+}(2 K)^{2}$, while when $n+r$ is odd we find that $I^{B}$ is most simply expressed as a polynomial in $g=\gamma_{-}(K)^{2}$. Introducing the notation $g_{j}=g^{w_{j}}$ and the fundamental step-operators from ref. [6],

$$
t_{L_{j}}=t_{L_{j}}^{t}=\sum_{l=-L_{j} / 2}^{L_{j} / 2} g_{j}^{l}
$$

it is possible to prove [6] that the nasty looking expression for $I^{B e}$ displayed above reduces to:

$$
I_{L \widetilde{L}}^{B}=\prod_{j} n_{L_{j} \widetilde{L}_{j}} \quad \text { with } \quad n_{L_{j} \widetilde{L}_{j}}=t_{L_{j}} t_{\widetilde{L}_{j}}\left(1-g_{j}^{-1}\right) .
$$

We find exactly the same form also for the odd case, even if the representation involving $\gamma_{-}$is used. The particular representation of the intersection form chosen here emerges naturally from the BCFT, through the 'doubling trick' for fusion coefficients described above, but it is highly redundant. There are many non-trivial relations between the set of boundary states ||$L M S\rangle\rangle_{B}$, and this is reflected in the redundancy encoded in $I^{B}$. Similarly, the boundary state itself is redundantly labelled by $M$, or equivalently the $U(1)$ charges $q_{j}=M_{j} / h$ of the minimal model states from which it is built. For the spinless sector we write: $\left.\left.\| q\rangle\rangle_{B}=\| L=0 ; q\right\rangle\right\rangle_{B}$, while higher spin states (not all independent) are again given by the step-operators: $\left.\left.\| L ; q\rangle\rangle_{B}=\prod_{j} t_{L_{j}}|| q\right\rangle\right\rangle_{B}$.

The number of moduli of these boundary states are also given by the (extended) fusion coefficients [3]

$$
\mu_{L}^{B}=\frac{1}{2} \prod_{j}\left|n_{L_{j} L_{j}}(|g|)\right|-\nu
$$

where subtracting $\nu$ compensates for the counting of vacuum states. If $n+r$ is odd, $\nu=2^{\ell}$, where $\ell$ is the number of $L_{j}$ 's equal to $k_{j} / 2$ [6]. However, if $n+r$ is even, we find that $\nu=2^{\ell-1}$ when $\ell>0$, and $\nu=1$ if $\ell=0$. Hence if all $k_{j}$ are odd, $\nu$ is always equal to one.

Note that from a CFT point of view we are free to extend the tensor product with trivial $\left(k_{j}=0\right)$ factors, if we so desire. Thus, although $\gamma_{-}(K)^{2}$ provides the simplest and most obvious representation when $n+r$ is odd, we can also work in the 
$n+r+1$ even channel where computing the intersection form and number of moduli requires appending a factor of $P_{ \pm}(g)=\left(1 \pm g^{K / 2}\right) / 2$, appropriate for a minimal model with no central charge. Since $P_{+}(g)$ and $P_{-}(g)$ are (orthogonal) projection operators the number of such factors is immaterial. This appears to be the reason why the calculations reported in [6] are correct. As already explained, it is the even channel which offers the most direct down-link to geometry. It is a useful consistency check to compare results from computations in both channels, and we indeed found agreement.

We now want to transport the CFT data, in particular the charges of the B-type boundary states, to large radius points on the boundary of moduli space, where they can be compared with the Ramond charges of $D$-branes wrapping cycles of large CY manifolds. This can not be done without specifying some geometric data which select a CY space.

\section{Bundle data and brane charges}

We summarize the relevant $D$-brane geometry for the $K 3$-fibrations under examination here. $\mathbb{P}(1,1,2,2,2)[8]$ has been analyzed in great detail using mirror symmetry in ref. [12], who also discussed $\mathbb{P}(1,1,2,2,6)[12]$ in somewhat less detail; further explicit properties of the latter were worked out in ref. [13]. For each of the two compactifications the $N=2$ special geometry is encoded in a prepotential whose classical part can be written in the form:

$$
\mathcal{F}\left(t_{1}, t_{2}\right)=-\frac{1}{3 !} c_{111} t_{1}^{3}-\frac{1}{2} c_{112} t_{1}{ }^{2} t_{2}+\frac{1}{24}\left(b_{1} t_{1}+b_{2} t_{2}\right)+\text { const. }
$$

where $t_{1}$ and $t_{2}$ parametrize the complexified Kähler cone: $K=t_{1} J_{1}+t_{2} J_{2}$. Here $J_{1}$ and $J_{2}$ are the Kähler classes of the $K 3$-fiber and $\mathbb{P}_{1}$ base, respectively. The coefficients are given in terms of the following topological intersections:

$$
c_{i j k}=\int J_{i} \wedge J_{j} \wedge J_{k}, \quad b_{i}=\int c_{2}(X) \wedge J_{i}, i=1,2 .
$$

For the threefolds at hand, we have [12]:

$$
\begin{aligned}
\mathbb{P}(1,1,2,2,6)[12]: & c_{111}=4, c_{112}=2, b_{1}=52, b_{2}=24 \\
\mathbb{P}(1,1,2,2,2)[8]: & c_{111}=8, c_{112}=4, b_{1}=56, b_{2}=24
\end{aligned}
$$

$\dagger$ More precisely, $J_{1}=2 L+E$ and $J_{2}=L$, where $E$ is an exceptional divisor coming from blowing up a curve of singularities, and the linear system $|L|$ is a pencil of $K 3$ 's. 
From the prepotential one derives the symplectic period vector $\Pi \equiv\left(\mathcal{F}_{0} \equiv 2 \mathcal{F}-\right.$ $\left.t_{i} \partial_{i} \mathcal{F}, \mathcal{F}_{1}, \mathcal{F}_{2}, 1, t_{1}, t_{2}\right)^{t}$, which for $\mathbb{P}(1,1,2,2,6)[12]$ reads:

$$
\Pi=\left(\begin{array}{c}
\frac{13 t_{1}}{6}+\frac{2 t_{1}{ }^{3}}{3}+t_{2}+t_{1}{ }^{2} t_{2} \\
\frac{13}{6}-2 t_{1}{ }^{2}-2 t_{1} t_{2} \\
1-t_{1}{ }^{2} \\
1 \\
t_{1} \\
t_{2}
\end{array}\right)
$$

The entries (from the top down) correspond to integrations over $6-, 4-, 4-, 0-, 2-$ and 2-cycles, respectively.

We now want to obtain an explicit map between the topological invariants of $K$-theory (characteristic classes of the Chan-Paton sheaf $V$ ), and brane charges. The latter are defined as the coefficients of the $N=2$ central charge given by

$$
Z\left(n_{i}\right)=n_{6} \mathcal{F}_{0}+n_{4}^{1} \mathcal{F}_{1}+n_{4}^{2} \mathcal{F}_{2}+n_{0}+n_{2}^{1} t_{1}+n_{2}^{2} t_{2}
$$

Following ref. [6], the topological invariants can be obtained by comparing this to the central charge of a $D$-brane configuration

$$
Z(Q(V))=-\int e^{-K} \wedge Q=-\int e^{-K} \wedge \operatorname{Tr}\left(e^{F}\right) \wedge \sqrt{\widehat{A}(X)}
$$

where $Q=\left(r, c_{1}(V), \operatorname{ch}_{2}(V)+\frac{r}{24} c_{2}(X), \operatorname{ch}_{3}(V)+\frac{1}{24} c_{1}(V) c_{2}(X)\right) \in H^{2 *}(X)$ is the Mukai vector of effective brane charges [14,15]. Specifically, $r$ is the rank of the bundle and $\operatorname{ch}_{i}(V)$ are its Chern characters. Inserting the classical periods (11) and substituting the values of the intersections given in (10), equating (12) and (13) yields:

$$
\begin{aligned}
r & =n_{6}, \quad c_{1}(V)=\frac{1}{2} n_{4}^{1} J_{1}+\left(n_{4}^{2}-n_{4}^{1}\right) J_{2} \\
\operatorname{ch}_{2}(V) & =n_{2}^{1} h+n_{2}^{2} \ell, \quad \operatorname{ch}_{3}(V)=-n_{0}-\frac{1}{3} n_{4}^{1}-2 n_{4}^{2} .
\end{aligned}
$$

where $h=\frac{1}{2} J_{1} \wedge J_{2}$ and $\ell=\frac{1}{2} J_{1} \wedge J_{1}-J_{1} \wedge J_{2}$.

We will be mainly interested in $D$-branes wrapping the $K 3$-fiber (called "fiber branes" in ref. [15]). This corresponds to considering only states with $n_{4}^{1}=n_{2}^{2}=0$, and accordingly we will set $n_{4} \equiv n_{4}^{2}$ and $n_{2} \equiv n_{2}^{1}$. It follows from ref. [6] that the 
topological invariants of the torsion sheaf $V$ supported on the $K 3$-fiber can be written in terms of its extension $i_{*} V$ to the threefold as:

$$
Q=\left(0, r J_{2}, i_{*} c_{1}(V), i_{*} \operatorname{ch}_{2}(V)+r\right)
$$

That the vector of induced charges takes this naive form is a consequence of the triviality of the normal bundle, $c_{1}(K 3)=0$, and $c_{2}(K 3)=24$; otherwise, there would be extra correction terms. Comparing the central charges as before, we thus obtain a simple truncation of (14):

$$
r=n_{4}, \quad i_{*} c_{1}(V)=n_{2} h, \quad i_{*} \operatorname{ch}_{2}(V)=-n_{0}-2 n_{4} .
$$

We are particularly interested in the dimensions of the moduli spaces $\mathcal{M}$ of $D$-brane configurations on the $K 3$-fiber. Unlike for threefolds, for an isolated $K 3$ such dimensions (for irreducible bundles) are completely determined by the Mukai vector [16:

$$
\mu_{K 3}(Q) \equiv \operatorname{dim}_{c} \mathcal{M}(Q)=\langle Q, Q\rangle+2 .
$$

To write this in terms of the brane charges $n_{i}$, we need to know what the precise form of the inner product in (17) is. For this, note that the intersection form of the $K 3$-fiber is easily exhibited by transforming the periods (11) in the $t_{2} \rightarrow \infty$ limit to a more suitable basis, given by $\widetilde{\Pi}^{t}=\left(1, t_{1}, t_{1}^{2}, t_{2}, t_{2} t_{1}, t_{2} t_{1}{ }^{2}\right)^{t}$. The symplectic intersection form on the threefold then turns into $I^{\text {geom }} \rightarrow \widetilde{I}^{\text {geom }}=\left(\begin{array}{cc}0 & 1 \\ -1 & 0\end{array}\right) \otimes I_{K 3}$, where

$$
I_{K 3}=\left(\begin{array}{ccc}
0 & 0 & -1 \\
0 & \frac{1}{2} & 0 \\
-1 & 0 & 0
\end{array}\right) \text {. }
$$

Accordingly, the Mukai charge vector on $K 3$ takes the form $Q_{K 3}=\left(r, c_{1}(V), \operatorname{ch}_{2}(V)+\right.$ $r)=\left(n_{4}, n_{2},-n_{0}-n_{4}\right)$, whence:

$$
\mu_{K 3}\left(n_{i}\right)=2+\frac{1}{2} n_{2}^{2}+2 n_{0} n_{4}+2 n_{4}^{2}
$$

Analogously, for the threefold $\mathbb{P}(1,1,2,2,2)[8]$ we find that the intersection form on the fiber is half of the one given in (18), and $Q_{K 3}=\left(2 r, c_{1}(V), \operatorname{ch}_{2}(V)+r\right)=$ $\left(2 n_{4}, n_{2},-n_{0}-n_{4}\right)$. We therefore get in this case:

$$
\mu_{K 3}\left(n_{i}\right)=2+\frac{1}{4} n_{2}{ }^{2}+2 n_{0} n_{4}+2 n_{4}{ }^{2} .
$$




\section{Matching of geometric and BCFT data}

We now relate the BCFT data of Sect. 2 to the geometrical data of the previous section. This first of all involves an analytic continuation of the large-radius periods (11) to the Gepner point, i.e. $Z=\vec{n} \cdot \vec{\Pi}=\left(\vec{n}^{G} m^{-1}\right) \cdot\left(m \vec{\Pi}^{G}\right)$. The continuation matrix $m$ is path dependent and thus only defined up to $S p(6, \mathbb{Z})$ monodromy transformations. It is most natural to choose, similar to the case in refs. [12,6], a preferred basis such that the conifold singularity corresponds to a single wrapped D6-brane.

By applying the method for analytic continuation described in ref. [12] to the threefold $\mathbb{P}(1,1,2,2,6)[12]$, we find after some work that for our choice of periods (11) the continuation matrix takes the form

$$
m=\left(\begin{array}{cccccc}
-1 & 1 & 0 & 0 & 0 & 0 \\
\frac{3}{2} & \frac{3}{2} & \frac{1}{2} & \frac{1}{2} & -\frac{1}{2} & -\frac{1}{2} \\
1 & 0 & 1 & 0 & 0 & 0 \\
1 & 0 & 0 & 0 & 0 & 0 \\
-\frac{1}{2} & 0 & \frac{1}{2} & 0 & \frac{1}{2} & 0 \\
\frac{1}{2} & \frac{1}{2} & -\frac{1}{2} & \frac{1}{2} & -\frac{1}{2} & \frac{1}{2}
\end{array}\right)
$$

The geometric intersection form in the Gepner basis is therefore:

$$
I^{G}=m^{-1} I^{\text {geom }} m^{-1, t}=\left(\begin{array}{cccccc}
0 & 1 & 0 & -2 & 0 & 1 \\
-1 & 0 & 1 & 0 & -2 & 0 \\
0 & -1 & 0 & 1 & 0 & -2 \\
2 & 0 & -1 & 0 & 1 & 0 \\
0 & 2 & 0 & -1 & 0 & 1 \\
-1 & 0 & 2 & 0 & -1 & 0
\end{array}\right) .
$$

On the other hand, for $\mathbb{P}(1,1,2,2,2)[8]$ we get

$$
m=\left(\begin{array}{cccccc}
-1 & 1 & 0 & 0 & 0 & 0 \\
\frac{3}{2} & \frac{3}{2} & 0 & 0 & -\frac{1}{2} & -\frac{1}{2} \\
1 & 0 & 1 & 0 & 0 & 0 \\
1 & 0 & 0 & 0 & 0 & 0 \\
-\frac{1}{4} & 0 & \frac{1}{2} & 0 & \frac{1}{4} & 0 \\
\frac{1}{4} & \frac{3}{4} & -\frac{1}{2} & \frac{1}{2} & -\frac{1}{4} & \frac{1}{4}
\end{array}\right)
$$

and an expression for $I^{G}$ similar to (22).

$\dagger$ Note that we use a basis different to the one of ref. [12]; moreover, we found that there are some typing errors in their monodromy matrices. 
Since there is no way the BCFT can know how we will choose the continuation path for a given large-radius 'endpoint' threefold, the monodromy basis selected by the above construction of boundary states need not be the same as the Gepner basis associated with our choice of $m$. A further change of basis is necessary to link the BCFT boundary state charges to the geometrical charges at the Gepner point. The precise form of the required transformation can be determined by comparing the geometric intersection matrix at the Gepner point (22) with the projection of the $L_{i}=0$ BCFT intersection matrix (11), (3) onto the non-redundant basis.

In all cases studied in refs. [3, [6] and by us so far, it turns out that $I^{B}$ is to be compared with $(1-g) T I^{G} T^{t}(1-g)^{t}$, where $T$ is the appropriate intertwining matrix. Taking everything together, the geometric brane configurations $\left|n_{i}\right\rangle^{\infty}$ are given in terms of the boundary CFT charges by $\left.\left.\left|n_{i}\right\rangle^{\infty}=\left(m^{-1}\right)^{t} T^{t}(1-g)^{t}|| L ; q\right\rangle\right\rangle_{B}$, where $n_{i}$ can be thought of as functions of the BCFT labels $\{L ; q\}$.

What we find for the two models considered here are lists of charges that are similar to those of ref. [6]. Indeed a similar bundle interpretation can be given for some of them, however in order not to be repetitious, we refrain from presenting this here. There are also certain states, like the one with charges $(1,-2,0)$, for which there is no conventional bundle interpretation, and this is analogous to the findings of [3].

The major difference of our work and ref. [6] is that we deal with $K 3$-fibrations rather than elliptic fibrations. We will thus focus here on the brane features which are specific to $K 3$ fibrations, and in particular on brane configurations supported on the $K 3$ fiber. Tables 1 and 2 list all brane states we have found within this construction that have $n_{6}=n_{4}^{1}=n_{2}^{2}=0$, together with some of their essential characteristics. 


\begin{tabular}{|cc|ccc|cc|}
\hline$L_{i}$ & $\nu$ & \multicolumn{3}{|c|}{$\left(r, \mathrm{ch}_{2}(V), c_{1}(V)\right)$} & $\mu_{L}^{B}$ & $\Delta$ \\
\hline$[1,0,0,0]$ & 1 & $(1,0,0)$ & $(1,1,-2)$ & $(2,-1,-2)$ & 1 & 1 \\
\hline$[3,0,0,0]$ & 1 & $(1,-2,0)$ & $(1,-1,-2)$ & $(0,-1,2)$ & 5 & 1 \\
\hline$[3,0,1,0]$ & 1 & $(1,0,-4)$ & $(1,-3,2)$ & $(2,-3,-2)$ & 9 & 1 \\
\hline$[3,0,1,1]$ & 1 & $(3,-6,0)$ & $(3,-3,-6)$ & $(0,-3,6)$ & 21 & 1 \\
\hline$[5,0,0,0]$ & 2 & $(2,-2,0)$ & $(2,0,-4)$ & $(0,-2,0)$ & 6 & 4 \\
\hline$[5,0,1,0]$ & 2 & $(2,-4,0)$ & $(2,-2,-4)$ & $(0,-2,4)$ & 14 & 4 \\
\hline$[5,0,1,1]$ & 2 & $(2,0,-8)$ & $(2,-6,4)$ & $(4,-6,-4)$ & 30 & 4 \\
\hline$[5,0,2,0]$ & 4 & $(4,-4,-4)$ & $(0,-4,4)$ & $(0,0,4)$ & 20 & 10 \\
\hline$[5,0,2,1]$ & 4 & $(4,-8,0)$ & $(4,-4,-8)$ & $(0,-4,8)$ & 44 & 10 \\
\hline$[5,0,2,2]$ & 8 & $(4,0,-12)$ & $(4,-8,-4)$ & $(4,-8,4)$ & 64 & 22 \\
\hline
\end{tabular}

Table 1: CFT and bundle data of states localized on the $K 3$-fiber of $\mathbb{P}(1,1,2,2,6)[12]$. On the left we list the CFT labels $L_{i}$ and the number of vacuum states $\nu$, as defined in the text after eq. (7) (note that in some cases there are two sets of such labels that lead to the same states, and we show only one of them). In the middle we have three sets of charges, which have the same properties because they belong to the same $\mathbb{Z}_{12}$ orbit. On the right, $\mu_{L}^{B}$ is the number of BCFT moduli in the threefold as given by eq. (7). Finally, $\Delta \equiv \mu_{L}^{B}-\mu_{K 3}$ is the excess number of moduli of the boundary state as compared to the number of $K 3$ bundle moduli $\mu_{K 3}$ given by eq.(19).

\begin{tabular}{|c|c|c|c|c|}
\hline$L_{i}$ & \multicolumn{3}{|c|}{$\left(r, \operatorname{ch}_{2}(V), c_{1}(V)\right)$} & $\mu_{L}^{B} \quad \Delta$ \\
\hline$[1,0,0,0,0] \quad 1$ & \begin{tabular}{l|l}
1 & $(1,0,0)$
\end{tabular} & $(3,0,-8)$ & $(1,2,-4) \quad(3,-2,-4)$ & 1 \\
\hline$[3,0,0,0,0] \quad 1$ & 1 & $(2,-2,-4)$ & $(0,-2,4)$ & 7 \\
\hline$[3,0,1,0,0] \quad 2$ & 2 & $(2,-4,0)$ & $(2,0,-8)$ & 144 \\
\hline$[3,0,1,1,0] \quad 4$ & 4 & $(4,-4,-8)$ & $(0,-4,8)$ & $28 \quad 10$ \\
\hline$[3,0,1,1,1] \quad 8$ & 8 & $(4,-8,0)$ & $(4,0,-16)$ & $56 \quad 22$ \\
\hline
\end{tabular}

Table 2: Analogous data as in Table 1 for D-branes wrapped on the K3-fiber of $\mathbb{P}(1,1,2,2,2)[8]$. 


\section{Discussion}

The data in Tables 1 and 2 provide support for the conjectured equivalence of boundary states and $D$-branes. We confine ourselves here to the following remarks.

i) The entries of each row belong to the same $\mathbb{Z}_{K}$ orbit (which also contains other states not localized only on the $K 3$ fiber that we don't show). They thus have the same properties with regard to the moduli space, even though the brane charges can be very different. All charges satisfy the BPS condition [15] $\left\langle Q_{K 3}, Q_{K 3}\right\rangle \geq-2$, with $r>0$ or $r=0, c_{1}>0$ or $r=c_{1}=0, \operatorname{ch}_{2}(V) \equiv \frac{1}{2} c_{1}^{2}-c_{2}<0$.

ii) Consider the charge vectors of the form $Q_{K 3}=\left(r,-c_{2}, 0\right)$, which can be interpreted as $S U(r)$ bundles with $c_{1}=0$. For such bundles $c_{2}$ must obey $c_{2} \geq 2 r$. We indeed find, as a first consistency test, that for all such charges, $c_{2}$ is equal to $2 r$, except for $r=2$ where we find in addition also $c_{2}=r$. However, this can be given an interpretation as an $S O(3)$ bundle instead of $S U(2)$, so all is well.

iii) More interestingly, note that for boundary states with one vacuum $(\nu=1$ in (7)), the number of CFT moduli is one larger than the number of classical $K 3$ moduli: $\Delta \equiv$ $\mu_{L}^{B}-\mu_{K 3}=1$. This is indeed exactly as expected from geometrical considerations: the brane system should gain a modulus from the one-dimensional base space $\left(\mathbb{P}_{1}\right.$ here) when it is embedded as a fiber in a threefold.

iv) On the other hand, if the number of CFT vacua gets larger, $\nu>1$, there is a growing discrepancy between the numbers of threefold BCFT moduli and classical $K 3$ moduli. However, as suggested in [6], such boundary states may describe not single branes but collections of several branes, and this would correspond to reducible bundles or sheaves. For such bundles the geometry of the moduli space is more intricate than for irreducible ones, as the relative positions of the configurations correspond to Coulomb branches of additional moduli [17].

By studying the tables one finds consistency between the charge vectors and the values of $\nu$. That is, configurations with $\nu>1$ can be decomposed intoconfigurations with smaller $\nu_{i}$, in a way such that $\nu_{i}$ and the charges add up correctly. ${ }^{t}$ For example, the configurations with charges $(2,-2 k, 0), k=1,2$ have $\nu=2$, and indeed can be decomposed [17] into two branes with charges $\left(1, \frac{1}{2} c_{1}^{2}(L), \pm c_{1}(L)\right)$. These correspond to reducible bundles of the form $L \oplus L^{-1}$, where $L$ are line bundles with $c_{1}^{2}(L)=-2 k$.

1 Note that $\operatorname{ch}_{2}(V)<0$ corresponds to an anti-selfdual gauge connection, and positive brane charges, cf. eq. (16).

$\dagger$ Details will be discussed elsewhere. 
The Higgs branches of these $U(1) \times U(1)$ theories happen to coincide [17 with the moduli spaces of $S U(2)$ bundles with charges $(2,-2 k, 0)$. This illustrates that the charge label alone does not completely specify the configuration, rather it is $\nu$ that tells that we have a reducible $U(1) \times U(1)$ bundle rather than an $S U(2)$ bundle (however, these configurations are continuously connected, so that a distinction is not fundamentally important.) In contrast, the configuration $(3,-6,0)$ in table 1 is irreducible because $\nu=1$. Therefore it corresponds to an $S U(3)$ bundle with no Coulomb branch.

Some interesting observations can also be made by comparing both tables: for a given number $\nu$ of CFT vacuum states, we find that the number of excess moduli $\Delta$ is always the same. For example, the state with charges $(4,-4,-8)$ has 44 CFT moduli on $\mathbb{P}(1,1,2,2,6)[12]$, which is 10 more than what the Mukai formula gives for the $K 3$ alone. On the other hand, it has 28 moduli on $\mathbb{P}(1,1,2,2,2)[8]$, which is again 10 more than one would have for the corresponding $K 3$.

The point is that while the absolute numbers of moduli may differ, the excess number of moduli coming from the fibration is universal and given by ${ }^{-1} \Delta=3 \nu-2$. This can be given the following interpretation. From index theorems we know that $\mu_{K 3}=N_{m}-2\left(N_{g}-1\right)$, where $N_{m}=2 r \int c_{2}$ is the number of matter fields in six dimensions and $N_{g}$ is the total number of gauge fields. For an irreducible bundle we have $N_{g}=\operatorname{dim} G+1$, where the shift by one is due to the $U(1)$ factor that corresponds to the overall center-of-mass modulus of the brane configuration [3]. For a reducible bundle, which corresponds to a collection of branes, we have several centerof-mass moduli and thus $N_{g}=\operatorname{dim} G+N_{U(1)}$, where $N_{U(1)}$ is equal to the number of irreducible components. Hence we can identify $N_{U(1)}=\nu$ and express the excess number of moduli as:

$$
\begin{aligned}
\Delta \equiv \mu_{L}^{B}-\mu_{K 3} & =\mu_{L}^{B}-\left(N_{m}-2\left(N_{g}-1\right)\right) \\
& =\left(\mu_{L}^{B}-\left(N_{m}-2 \operatorname{dim} G\right)\right)+2(\nu-1) \\
& =: \widetilde{\Delta}+2(\nu-1) .
\end{aligned}
$$

$\diamond$ Note that the two K3's have different intersection forms, so the Mukai formulas (19) and 20) yield different numbers of moduli.

$\ddagger$ This is a general property of the BCFT, as will be shown elsewhere. 
The second term may be thought of as a correction to the Mukai formula $\mu_{K 3}=$ $2 r \int c_{2}-2 \operatorname{dim} G$, taking into account that we have a reducible bundle if $\nu>1$. A comparison with the CFT result $\Delta=3 \nu-2$ then yields

$$
\widetilde{\Delta}=\sum_{\substack{\text { irreducible } \\ \text { components }}}^{\nu} \widetilde{\Delta}_{(i)}=\nu,
$$

which means that each irreducible component of the $K 3$ bundle or sheaf gains one extra modulus upon fibration - which is precisely as expected! This is because each such component corresponds to an independent brane configuration that can sit anywhere over the $\mathbb{P}^{1}$ base.

Concluding, we note that there is a high degree of consistency and universality in our results. We therefore believe that these provide a successful test of the BCFT approach to $D$-branes.

Our findings suggest in particular that the moduli space of the $K 3$ remains protected even when embedded in a threefold with less supersymmetries, and its dimension is still given by the Mukai formula (17), if we subtract the degrees of freedom associated with the fibration over the $\mathbb{P}_{1}$ base. While this robustness may not be too surprising in an adiabatic, large base limit of the threefold, it is more impressive here as we look into a region of the threefold moduli space (the Gepner point) where the classical geometry is maximally distorted by quantum corrections. It appears that nothing serious happens to the branes supported on the $K 3$-fiber at large radius when we transport them all the way to the Gepner point of the CY moduli space. One may argue that there is an $N=4$ subsector in the $N=2$ BCFT that protects these states from instanton corrections also in the non-geometric regime.

\section{Acknowledgements}

We would like to thank Jürg Fröhlich, Jürgen Fuchs, Michael Gutperle, Graham Ross, Christoph Schweigert, and especially Peter Mayr for useful discussions, and Emanuel Diaconescu for illuminating correspondence. 


\section{References}

[1] H. Ooguri, Y. Oz and Z. Yin, D-branes on Calabi-Yau spaces and their mirrors, Nucl. Phys. B477 407 (1996), hep-th/9606112.

[2] C. Vafa, Extending mirror conjecture to Calabi-Yau with bundles, hep-th/9804131.

[3] I. Brunner, M.R. Douglas, A. Lawrence and C. Römelsberger, D-branes on the quintic, hep-th/9906200.

[4] M.R. Douglas, Topics in D-geometry, hep-th/9910170.

[5] D. Diaconescu and J. Gomis, Fractional branes and boundary states in orbifold theories, hep-th/9906242.

[6] D. Diaconescu and C. Römelsberger, D-branes and bundles on elliptic fibrations, hep-th/9910172.

[7] A. Recknagel and V. Schomerus, D-branes in Gepner models, Nucl. Phys. B531 185 (1998), hep-th/9712186.

[8] J. Fuchs and C. Schweigert, Branes: From free fields to general backgrounds, Nucl. Phys. B530 99 (1998), hep-th/9712257.

[9] M. Gutperle and Y. Satoh, D-branes in Gepner models and supersymmetry, Nucl. Phys. B543 73 (1999), hep-th/9808080; D0-branes in Gepner models and N = 2 black holes, Nucl. Phys. B555 477 (1999), hep-th/9902120.

[10] S. Govindarajan, T. Jayaraman and T. Sarkar, World sheet approaches to Dbranes on supersymmetric cycles, hep-th/9907131.

[11] A. Klemm, W. Lerche and P. Mayr, K3 Fibrations and heterotic type II string duality, Phys. Lett. B357 313 (1995), hep-th/9506112;

P. S. Aspinwall and J. Louis, On the Ubiquity of K3 Fibrations in String Duality, Phys. Lett. B369 233 (1996), hep-th/9510234.

[12] P. Candelas, X. De La Ossa, A. Font, S. Katz and D.R. Morrison, Mirror symmetry for two parameter models. I, Nucl. Phys. B416 481 (1994), hep-th/9308083.

[13] S. Kachru, A. Klemm, W. Lerche, P. Mayr and C. Vafa, Nonperturbative results on the point particle limit of $N=2$ heterotic string compactifications, Nucl. Phys. B459 537 (1996), hep-th/9508155.

[14] M. B. Green, J. A. Harvey and G. Moore, I-brane inflow and anomalous couplings on D-branes, Class. Quant. Grav. 1447 (1997), hep-th/9605033. 
[15] J.A. Harvey and G. Moore, On the algebras of BPS states, Commun. Math. Phys. 197489 (1998), hep-th/9609017.

[16] S. Mukai, Symplectic structure of the moduli of sheaves on an abelian or K3 surface, Invent. Math. 77(1984) 101; On the moduli space of bundles on K3 surfaces, I in Vector Bundles on Algebraic Varieties, Tata Inst. of Fund. Research.

[17] M. Bershadsky and V. Sadov, F-theory on K3 x K3 and instantons on 7-branes, Nucl. Phys. B510 232 (1998), hep-th/9703194. 